\title{
3D Plasmonic Ensembles of Graphene Oxide and Nobel Metal Nanoparticles with Ultrahigh SERS Activity and Sensitivity
}

\author{
Jing Lin, ${ }^{1,2}$ Xiansong Wang, ${ }^{3}$ Guangxia Shen, ${ }^{1}$ and Daxiang Cui ${ }^{1}$ \\ ${ }^{1}$ Institute of Nano Biomedicine and Engineering, Shanghai Jiao Tong University, 800 Dongchuan Road, Shanghai 200240, China \\ ${ }^{2}$ Guangdong Key Laboratory for Biomedical Measurements and Ultrasound Imaging, School of Biomedical Engineering, \\ Shenzhen University, Shenzhen 518060, China \\ ${ }^{3}$ Department of Plastic and Reconstructive Surgery, Shanghai Key Laboratory of Tissue Engineering, Shanghai Ninth People's Hospital, \\ Shanghai Jiao Tong University School of Medicine, Shanghai 200011, China
}

Correspondence should be addressed to Jing Lin; jingl@szu.edu.cn and Daxiang Cui; dxcui@sjtu.edu.cn

Received 24 January 2016; Accepted 6 April 2016

Academic Editor: Giuseppe Compagnini

Copyright (C) 2016 Jing Lin et al. This is an open access article distributed under the Creative Commons Attribution License, which permits unrestricted use, distribution, and reproduction in any medium, provided the original work is properly cited.

\begin{abstract}
We describe a comparison study on 3D ensembles of graphene oxide (GO) and metal nanoparticles (silver nanoparticles (AgNPs), gold nanoparticles (GNPs), and gold nanorods (GNRs)) for surface-enhanced Raman scattering (SERS) application. For the first time, GNRs were successfully assembled on the surfaces of GO by means of electrostatic interactions without adding any surfactant. The SERS properties of GO/AgNPs, GO/GNPs, and GO/GNRs were compared using 2-mercaptopyridine (2-Mpy) as probing molecule. We found that GO/AgNPs and GO/GNPs substrates are not suitable for detecting 2-Mpy due to the very strong $\pi$ - $\pi$ stacking interaction between the 2-Mpy molecules and $s p^{2}$ carbon structure of GO. Conversely, the GO/GNRs substrates show ultrahigh SERS activity and sensitivity of 2-Mpy with the detection limit as low as $\sim 10^{-15} \mathrm{M}$, which is $\sim 2-3$ orders of magnitude higher than that of the corresponding GNRs.
\end{abstract}

\section{Introduction}

Design and construction of novel assembly architectures with individual nanoparticles (NPs) promote the practical application of NPs into functional nano/microdevices [1-5]. Generally, assemblies exhibit new and unique collective properties that are different from those of both themselves and bulk counterparts [6]. Particularly, ensembles of noble-metal NPs (gold and silver), due to their unique plasmon resonance, have attracted increasing attention in optical waveguides [7], photothermal therapy [8, 9], surface-enhanced Raman scattering (SERS) [10, 11], and so on.

SERS is a fascinating and potentially analytical technique to detect a wide variety of biochemicals at trace even down to single-molecular level $[12,13]$. For individual noble-metal NPs (gold and silver), due to collective oscillation of conduction band electrons, when they are irradiated at the wavelength of their localized surface plasmon resonance (LSPR) peak, greatly enhanced electric $(E)$ field area will produce in special surface confined locations (namely, hotspots) such as the tips of gold nanorods (GNRs) and gold nanostars [14, 15]. Raman scattering signals of biochemicals within the vicinity of hotspots are dramatically amplified with $E^{4}$ dependence, which is called SERS. However, for individual noble-metal NPs (gold and silver), the uncontrolled aggregation directly decide the numbered and uniformly distributed on the surface of topographically complex [16, 17]. Therefore, development of novel SERS substrates with a large number of hotspots is still challenging for ultrasensitive biochemical detection.

Anisotropic GNRs with hotspots at their tips show better performance in Raman detection than spherical gold nanoparticles [18, 19]. Recently, assembly of GNRs has shown great potential to increase high density of hotspots, as a result of side-to-side, tip-to-tip, and side-to-tip plasmon coupling between adjacent GNRs [18, 20]. Yu group developed the self-assembly of GNRs in PVA nanofibers by electrospinning, which exhibited high SERS activity and sensitivity of Raman probe $3,3^{\prime}$-diethylthiatricarbocyanine iodide (DTTCI) with the trace level $10^{-7} \mathrm{M}$ [18]. Mirkin group have successfully 
prepared tailorable GNR dimers in high yield and high monodispersity ( $96 \%$ dimers) using on-wire lithography (OWL) method for SERS detection [16]. Afterwards, the GNR dimers were physically attached on the micrometer-sized, ultrathin, and flexible silica sheets [17]. Coupled with the ability to tailor the optical properties of each dimer and the unique ability of the nanosheets to conform to complex topographies while maintaining the geometry and integrity of the dimers, the novel nanosheets promise SERS application. However, those methods are complex and time-consuming and of high cost.

Graphene oxide (GO) is an atomically ultrathin and flexible sheet with large specific surface area [21, 22]. The sheet structure promises to assemble metal nanoparticles on two accessible sides of GO to build new 3D ensembles [2325]. Herein, we constructed three different 3D ensembles of metal nanoparticles (silver nanoparticles (AgNPs), gold nanoparticles (GNPs), and gold nanorods (GNRs)) on the surface of GO and conducted a comparison study of SERS application on them. The GO/AgNPs was synthesized by the in situ growth method. The GO/GNPs and GO/GNRs were prepared by the electrostatic self-assembly method. Their SERS properties were compared using 2-mercaptopyridine (2-Mpy) as probing molecule.

\section{Experimental Details}

2.1. Synthesis of GO. Water soluble GO was prepared by oxidizing graphite powder according to a modified Hummers' method [26] and our previous reports [21, 27, 28]. The aqueous solution of the GO $(0.5 \mathrm{mg} / \mathrm{mL})$ was stored at room temperature for further use.

2.2. Synthesis of GO/AgNPs. The GO/AgNPs was synthesized by the in situ growth method [27]. The aqueous solution of $\mathrm{AgNO}_{3}(10 \mathrm{mM})$ was gradually added to $5 \mathrm{~mL}$ of an aqueous solution of GO $(0.5 \mathrm{mg} / \mathrm{mL})$, by $1 \mathrm{~mL}$ per time for five times, while under vigorous stirring at room temperature for $10 \mathrm{~min}$. Then the mixed reaction solution was kept static under ambient conditions for aging.

2.3. Synthesis of GNPs and GO/GNPs. GNPs with a diameter of $20 \mathrm{~nm}$ were prepared by the use of citrate to reduce chloroauric acid in the aqueous phase $[8,9]$. A certain of PEI solution $(50 \mathrm{mg} / \mathrm{mL})$ was slowly added to a GO solution $(0.5 \mathrm{mg} / \mathrm{mL})$ to give a final weight ratio of GO : PEI $2: 1$. The mixture was sonicated for over $30 \mathrm{~min}$ and stirred overnight. The excess PEI molecules were removed by centrifugation filtration through $100 \mathrm{kDa}$ MWCO Amicon filters and washed away with water for several times with distilled water. Then $25 \mathrm{~mL}$ of GO-PEI aqueous solution was added to $1 \mathrm{~mL}$ of GNPs solution and incubated for $12 \mathrm{~h}$. The GO/GNPs composite was collected by centrifugation and stored at room temperature for further characterization and use.

2.4. Synthesis of GNRs and GO/GNRs. GNRs were synthesized via a seed-mediated growth method $[29,30]$. Before the self-assembly, the GNRs were purified by twice centrifugation at $9600 \mathrm{rpm}$ for $15 \mathrm{~min}$ to remove the excess CTAB molecules during the synthesis of GNRs. The GNRs $(0.5 \mathrm{mM})$ were dispersed in distilled water and stored at room temperature for further use.

For the self-assembly of GO and GNRs, a series of mass ratio of GO and GNRs from $1: 1$ to $1: 50$ was tried in this study. A certain volume of GO $(0.50 \mathrm{mg} / \mathrm{mL})$ was added to $1 \mathrm{~mL}$ of the GNRs aqueous solution $(0.5 \mathrm{mM})$ with vigorous shake. Then the mixed solution was sonicated for $1 \mathrm{~min}$. After $24 \mathrm{~h}$ incubation, the GO/GNRs composite was collected by centrifugation and stored at room temperature for further characterization and use.

2.5. SERS Experiments. All the experiments were carried out at room temperature. A series of 2-mercaptopyridine (2Mpy) aqueous solutions with different concentrations were prepared for SERS detection. $100 \mu \mathrm{L}$ of 2-Mpy was added to $200 \mu \mathrm{L}$ of GO/GNRs in a centrifugal tube and kept for $5 \mathrm{~h}$ to reach adsorption equilibrium under ultrasound. The 2-Mpy adsorbed GO/GNRs were washed for several times with distilled water and dispersed in $100 \mu \mathrm{L}$ of distilled water. The purified 2-Mpy adsorbed GO/GNRs aqueous solution was dropped onto a Si wafer. After evaporation of water, the Raman spectra of samples were measured.

2.6. Characterization. Transmission electron microscopy (TEM) images were taken by JEM-2010 (JEOL Ltd., Japan) operated at an accelerating voltage of $200 \mathrm{kV}$. Scanning electron microscopy (SEM) images were acquired on a field emission scanning electron microscope (FESEM, Zeiss Ultra). UV-Vis spectra were measured at $20^{\circ} \mathrm{C}$ using a Varian Cary 50 equipped with a $10 \mathrm{~mm}$ quartz cell, where the light path length was $1 \mathrm{~cm}$. Zeta potential measurements were measured by NICOMP 380ZLS Zeta potential/particle sizer. The Fourier transform infrared (FTIR) spectra were recorded on a Bruker EQUINOX 55 FTIR Spectrometer. Raman spectra were recorded by using Renishaw-1000-type confocal Raman spectrometer equipped with excitation source of the aircooled argon ion laser (Spectra-Physics Model 163-C4260). The excitation wavelength of $532 \mathrm{~nm}$ was used for GO/AgNPs and GO/GNPs samples. The excitation wavelength of $785 \mathrm{~nm}$ was used for GO/GNRs samples. The laser power of ca. $1.0 \mathrm{~mW}$ was used as the excitation source for the Raman experiments. Each spectrum was obtained using five accumulations, and the acquisition time in each case was $20 \mathrm{~s}$. Data analysis was performed by a Renishaw v1.3 WIRE software.

\section{Results and Discussion}

Water soluble GO was synthesized by oxidizing graphite powder according to the modified Hummers method [21, 22, 26, 28]. Atomic force microscopy (AFM) image (Figure 1(a)) shows that the planar nature of GO sheets allows them to be in good contact with the flat mica substrate as well as the stacking of GO layers (white arrows). The thickness is about $0.8 \mathrm{~nm}$ in Figure 1(b), indicating the formation of the singlelayered $\mathrm{GO}$.

GO/AgNPs were synthesized by the in situ growth of silver nanoparticles on the surfaces of GO. As shown in Figures 2(a) and 2(b), AgNPs are distributed on two accessible surfaces of GO. The size of AgNPs is in the range 


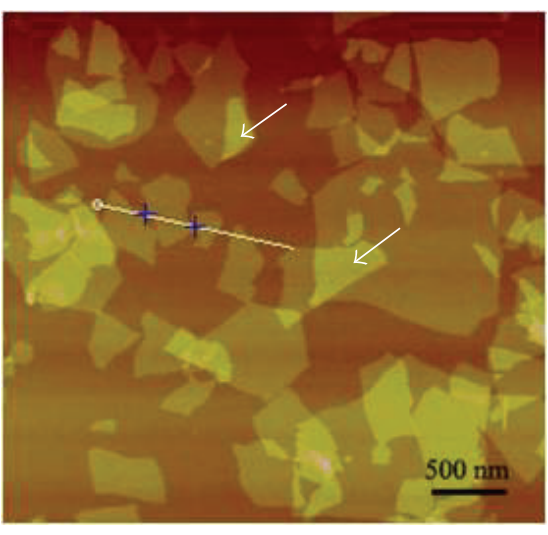

(a)

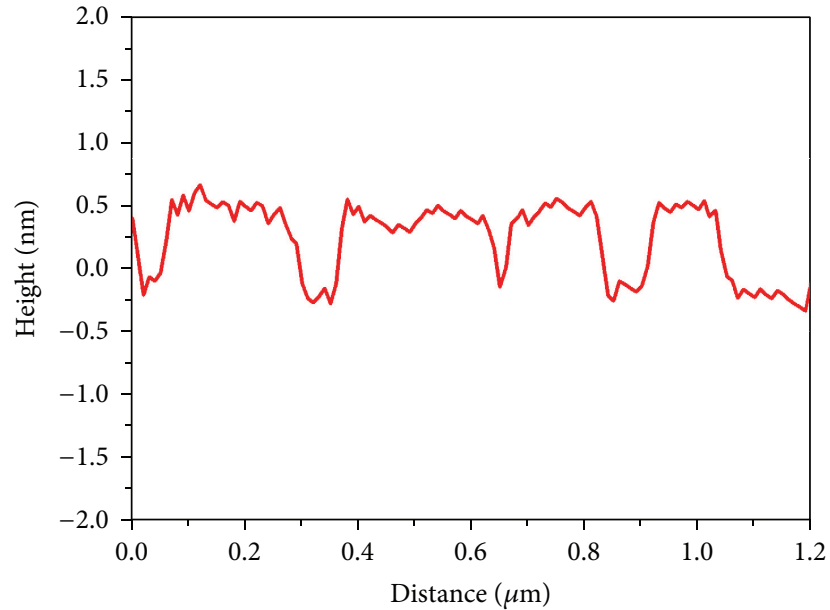

(b)

FIGURE 1: (a) High-resolution AFM imaging of single-layered graphene oxide (GO) nanosheets absorbed on a mica substrate. White arrows indicate the stacking of GO layers. (b) The height profile of the AFM image.

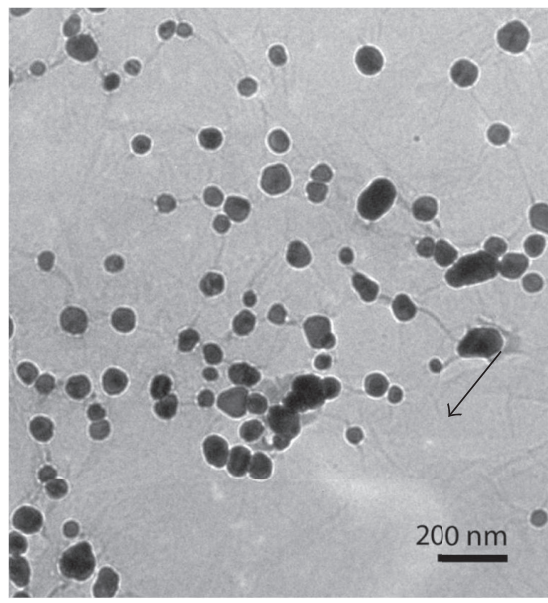

(a)

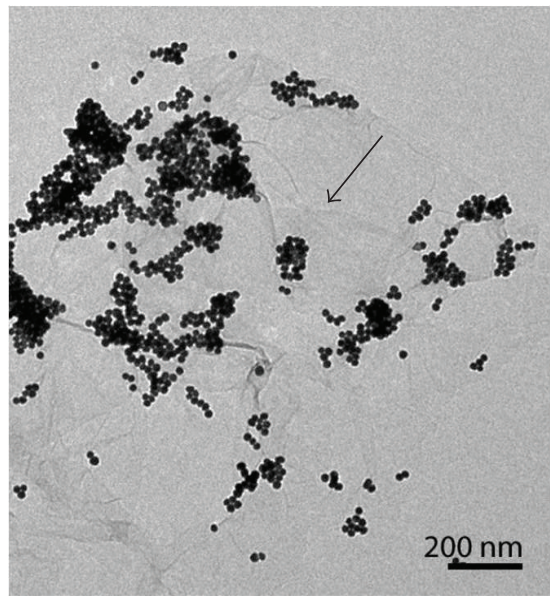

(c)

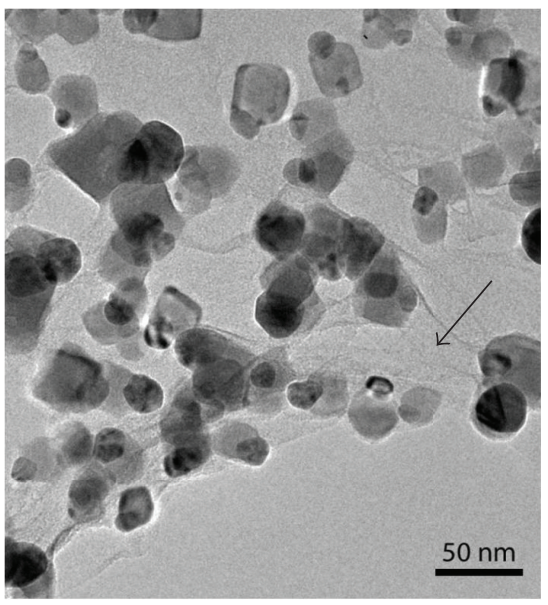

(b)

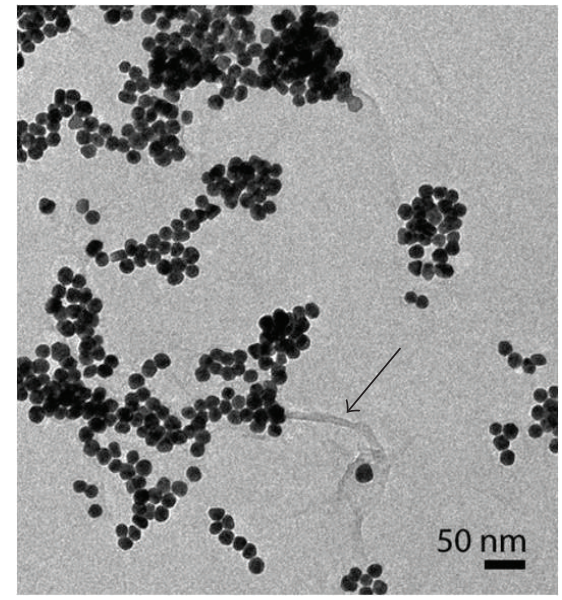

(d)

Figure 2: ((a), (b)) TEM images of GO/AgNPs. ((c), (d)) TEM images of GO/GNPs. Black arrows indicate the GO nanosheets. 


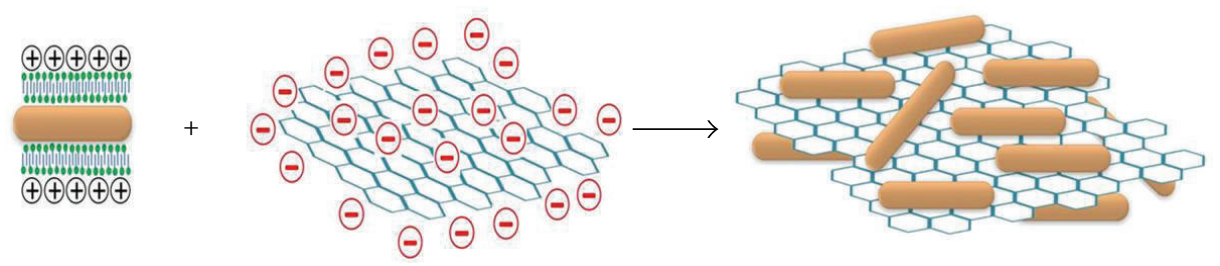

(a)

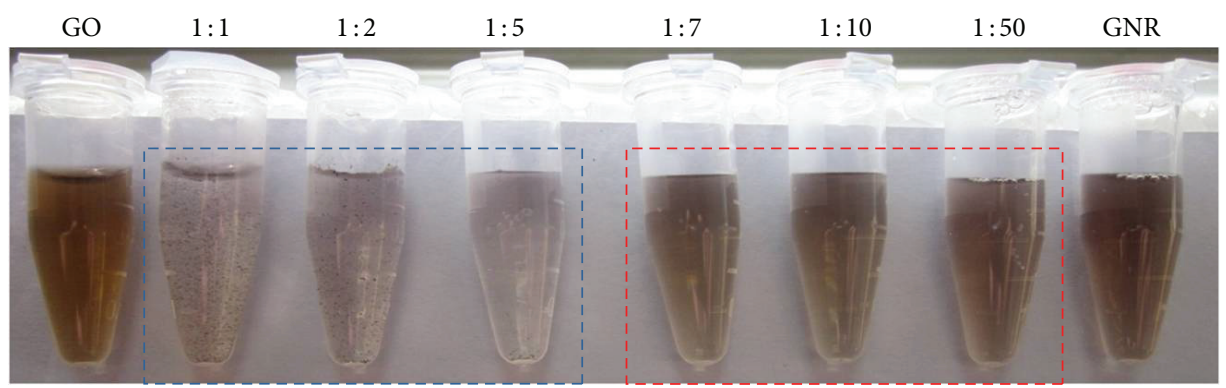

(b)

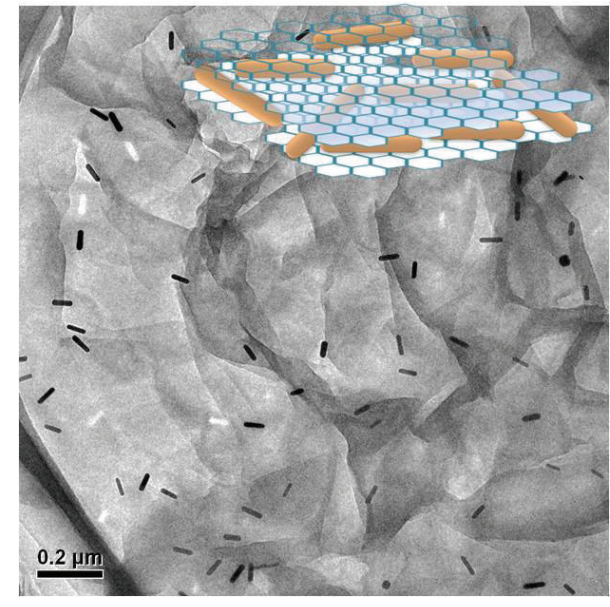

(c)

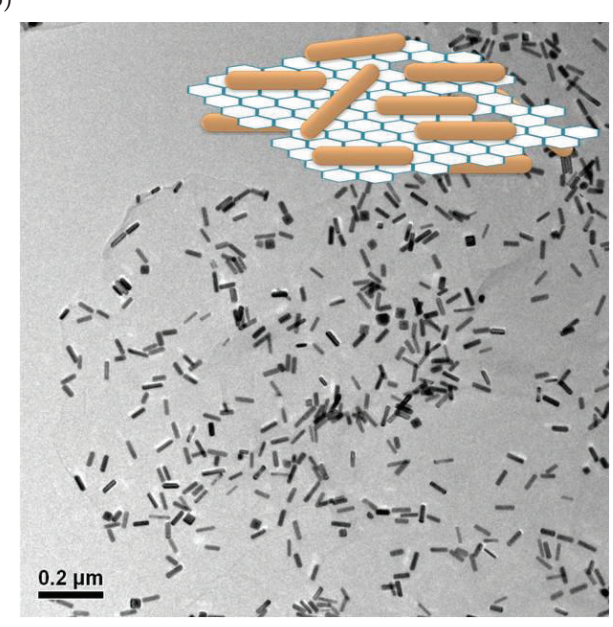

(d)

FIGURE 3: (a) Scheme illustrating the self-assembly of graphene oxide (GO) and gold nanorods (GNRs). CTAB-capped GNRs and GO sheets display positive and negative charge, respectively. (b) Photographs of GO/GNRs aqueous solutions with different mass ratio. Blue box: a lot of aggregates in the solutions. Red box: no aggregates in the solutions. (c) TEM image of GO/GNRs at the mass ratio of 1:2 (Inset: GO-GNRs-GO sandwich). (d) TEM image of GO/GNRs at the mass ratio of 1:10 (Inset: GNRs-GO-GNRs sandwich).

of $20 \sim 30 \mathrm{~nm}$. GO/GNPs were prepared by the assembly of negatively charged GNPs on the surfaces of positively charged polyethylenimine (PEI) modified GO. Figures 2(c) and 2(d) show GNPs with diameter of $20 \mathrm{~nm}$ distributed on two accessible surfaces of GO. GNRs with LSPR peak at $770 \mathrm{~nm}$ were prepared by the seed-mediated template-assisted protocol [29-31]. Before the self-assembly, the GNRs were purified by twice centrifugation at $9600 \mathrm{rpm}$ for $15 \mathrm{~min}$ to remove the excess CTAB molecules during the synthesis of GNRs. Figure 3(a) shows the self-assembly mechanism of negatively charged GO and positively charged GNRs. To find the best mass ratio of GO and GNRs for self-assembly, a series of mass ratio from $1: 1$ to $1: 50$ was tried in this study. As shown in Figure 3(b), when the ratio is less than 1:5, a lot of aggregates were found in the solutions. When the ratio is over $1: 7$, the products display excellent water solubility and stability. No aggregate has been found in the solutions even stored several weeks later. The color of GO/GNRs solution shows a GO-concentration dependent change from deep to light. The representative TEM images shown in Figures 3(c) and 3(d) display GO/GNRs having two different sandwich structures: GO-GNRs-GO at the mass ratio of $1: 2$ and GNRs-GO-GNRs at the mass ratio of $1: 10$. The results indicated that the assembly strongly depended on the mass ratio of GO and GNRs.

Since the density of hotspots on the GO sheet is dependent on the number of GNRs and their junctions, the proper mass of GNRs in GO/GNRs is crucial for SERS application. Two mass ratios (1:7 and 1:10) of GO and GNRs were carefully investigated by SEM and TEM. Figures 4(a)-4(c) show the SEM images of GO/GNRs complex at mass ratio 1:7. GNRs are evenly distributed on the two accessible surfaces of GO via electrostatic interactions, with almost no free GNRs in the SEM observation. With the increase of the ratio from 1:7 to $1: 10$ (Figures $4(\mathrm{~d})$ and $4(\mathrm{e})$ ), several free GNRs were 


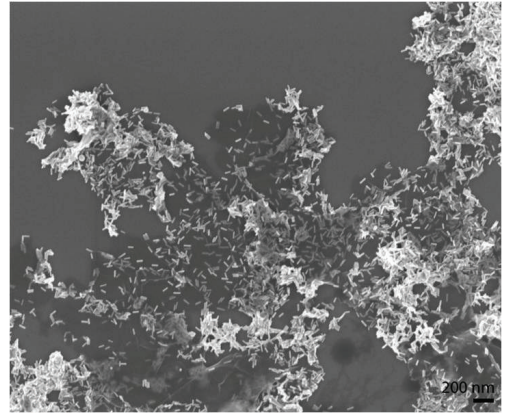

(a)

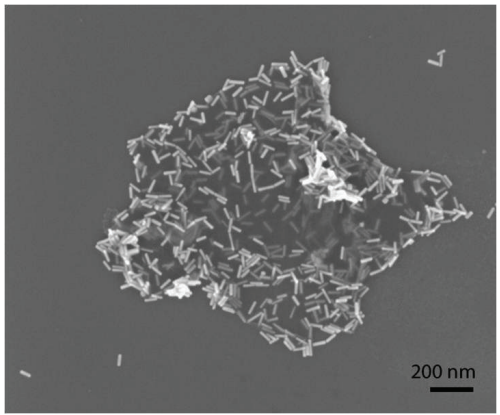

(d)

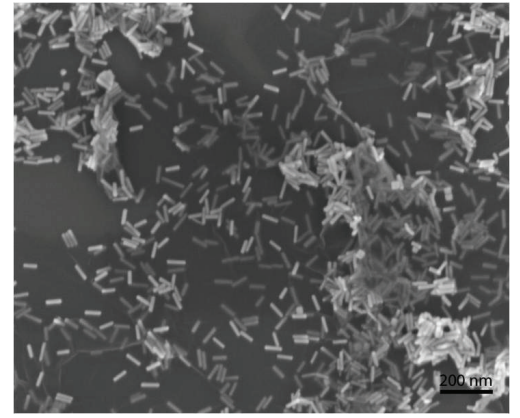

(b)

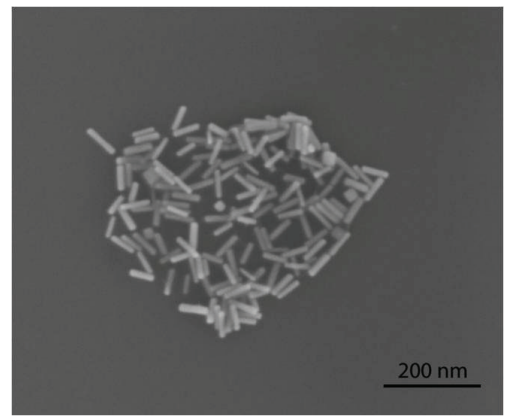

(e)

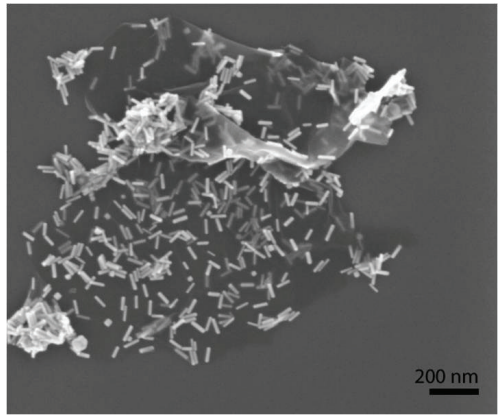

(c)

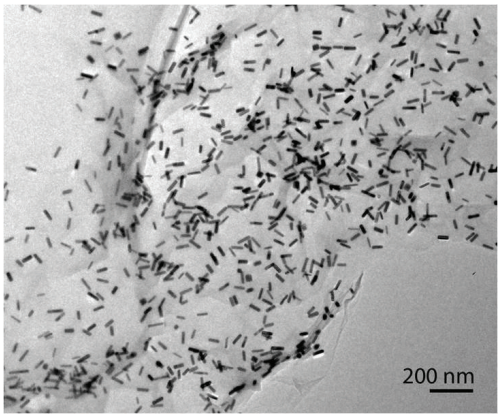

(f)

FIGURE 4: SEM images of GO/GNRs complex at different mass ratio of GO and GNRs. ((a)-(c)) 1:7 and ((d), (e)) 1:10. (f) TEM image of GO/GNRs complex at mass ratio $1: 10$.

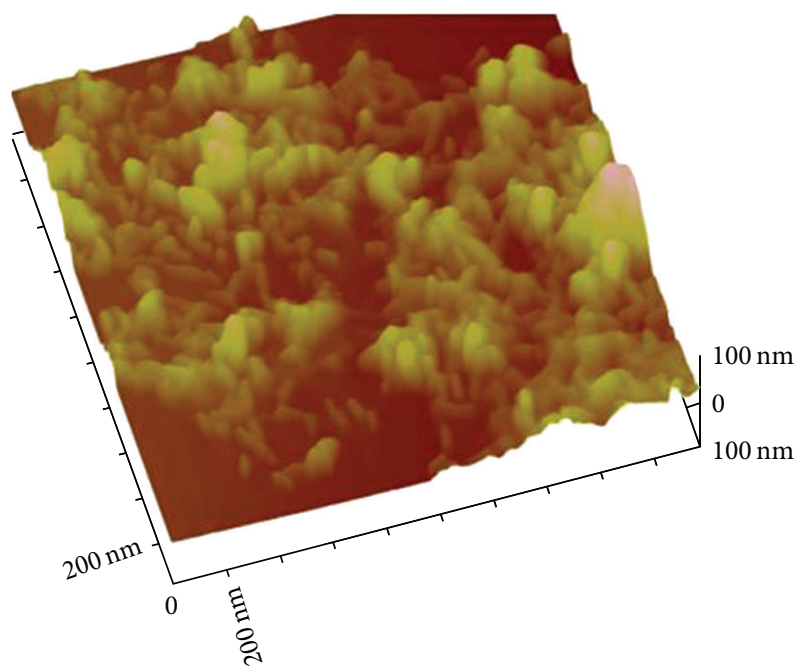

FIGURE 5: 3D AFM image of GO/GNRs complex at mass ratio 1:10.

found out of GO. The density of GNRs on the surfaces of GO/GNRs $(1: 10)$ is markedly higher than that of GO/GNRs $(1: 7)$. As shown in Figure 4(f), TEM image of GO/GNRs $(1: 10)$ also shows the similar results with SEM images. For the assembly of GO and GNRs at 1:10, the GNRs are a little bit excess, which suggested that the surfaces of GO were totally covered by GNRs. The 3D topography of GO/GNRs (1:10) was also observed by AFM imaging (Figure 5). Therefore, the GO/ GNRs $(1: 10)$ composite was chosen for later Raman detection.

To further study the interaction between GO and GNRs, UV-vis-NIR spectrophotometer was used to monitor the SPR absorption change of GNRs, and zeta potential measurement was employed to detect the surface charge of the resultant products. Figure 6(a) shows the UV-vis-NIR spectra of GO/GNRs aqueous solutions with different mass ratio. Pure GNRs show both the transverse and the longitudinal plasmon resonance peaks at 512 and $770 \mathrm{~nm}$, respectively. After GNRs assembled on the surfaces of GO, the SPR absorbance of GNRs shows GO-concentration dependent decrease along with the color gradually fading until being colorless finally [32]. The results were consistent with the observation in Figure 3(b). The intensity of longitudinal SPR peak decreased more quickly than that of the transverse SPR peak, which is probably due to the fact that the area at long axis direction of GNRs is much larger than that at the short axis direction as well as more positive charges on the area at long axis direction of GNRs. Therefore, the long axis of GNRs was bound preferentially to the surface of GO, depending on the large area and more positive charges.

As shown in Figure 6(b), zeta potential of GO/GNRs shows GNRs-concentration dependent increase. GO exhibits a negative charge $(-81.33 \pm 3.23)$, due to the abundant presence of carboxyl and hydroxyl groups on their surface. GNRs display a positive charge (36.09 \pm 2.93$)$, which is attributed to a lot of positive CTAB molecules on their surface. With the increase of GNRs concentration, the zeta potential of the GO/GNRs composites becomes more positive due to the assembly of positively charged GNRs on the surface of GO.

To compare the efficiency of GO/GNRs as SERS substrate, GO/AgNPs and GO/GNPs composites were chosen as control (Figure 7). GO/AgNPs were synthesized by the in situ growth of silver nanoparticles on the surfaces of GO 

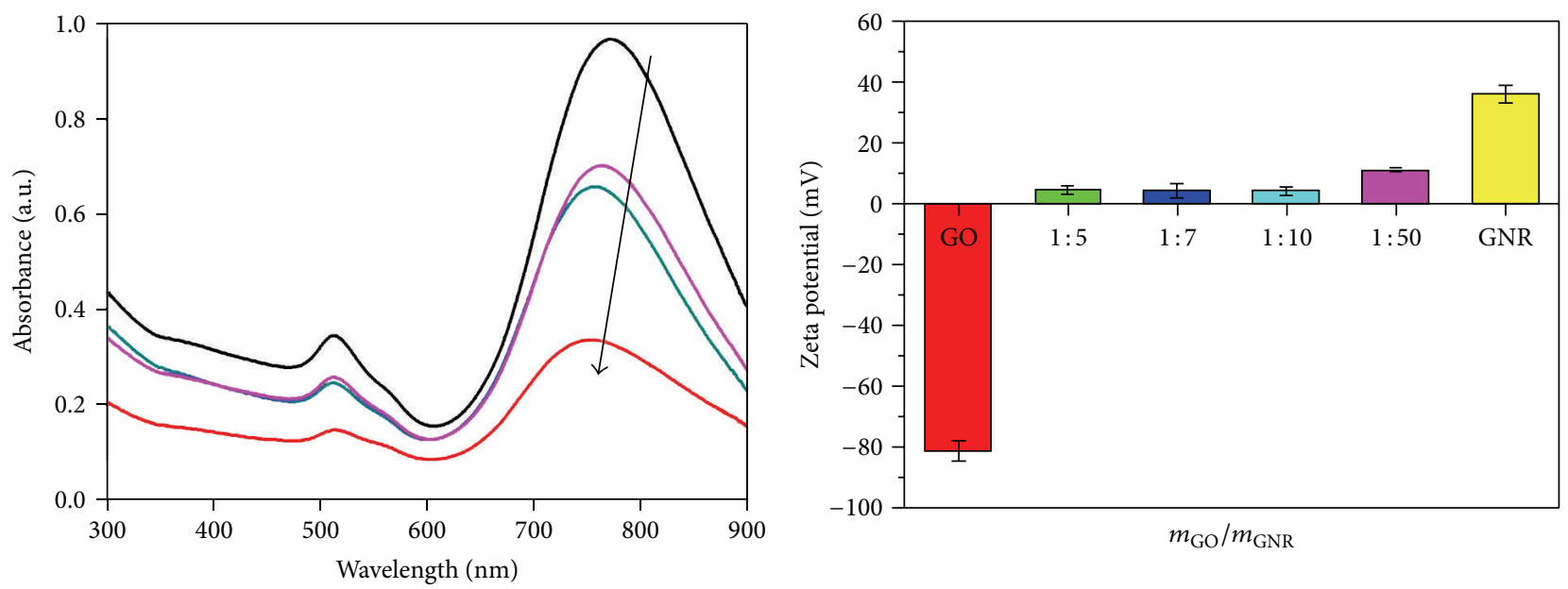

$\begin{array}{ll}-\mathrm{GO}: \mathrm{GNR}(0: 10) & -\mathrm{GO}: \mathrm{GNR}(1: 10) \\ \text { GO:GNR }(0.2: 10) & -\mathrm{GO}: \operatorname{GNR}(2: 10)\end{array}$

(a)

(b)

FIGURE 6: (a) UV-vis-NIR spectra of GO/GNRs aqueous solutions with different mass ratio. (b) Zeta potential of GO, GO/GNRs, and GNRs.

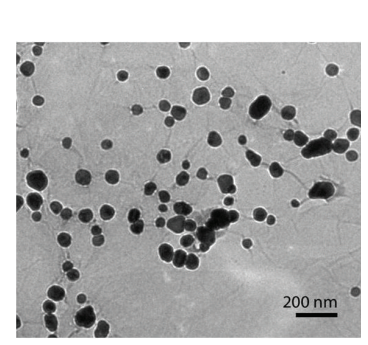

(a)

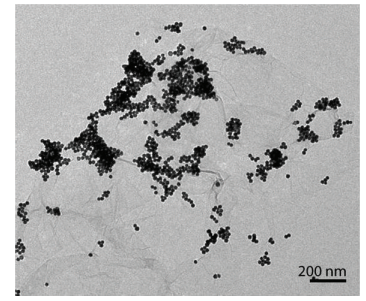

(c)

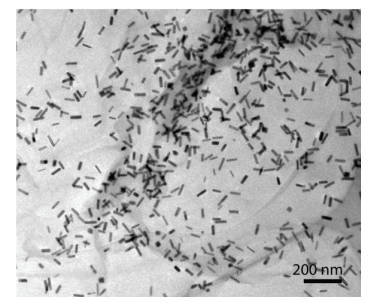

(e)
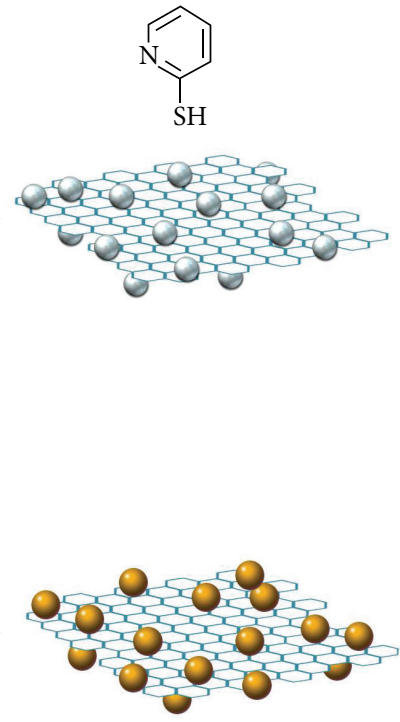

$\longrightarrow$

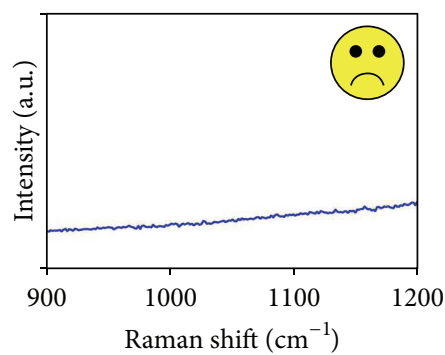

(d)

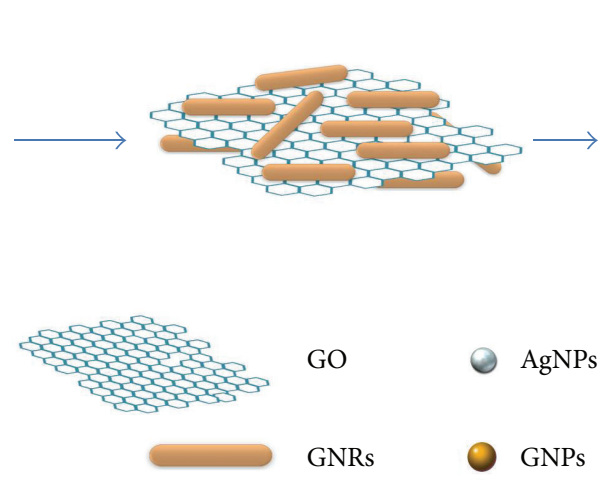

(f)

Figure 7: TEM images of GO/AgNPs (a), GO/GNPs (c), and GO/GNRs (e). SERS spectra of 2-mercaptopyridine $(2-\mathrm{Mpy})\left(1 \times 10^{-5} \mathrm{M}\right)$ at different substrates: GO/AgNPs (b), GO/GNPs (d), and GO/GNRs (f). 


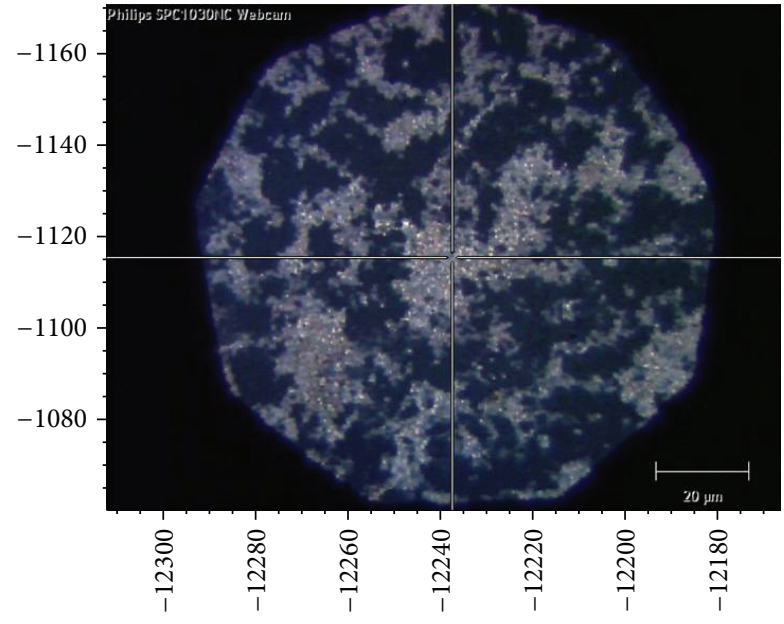

(a)

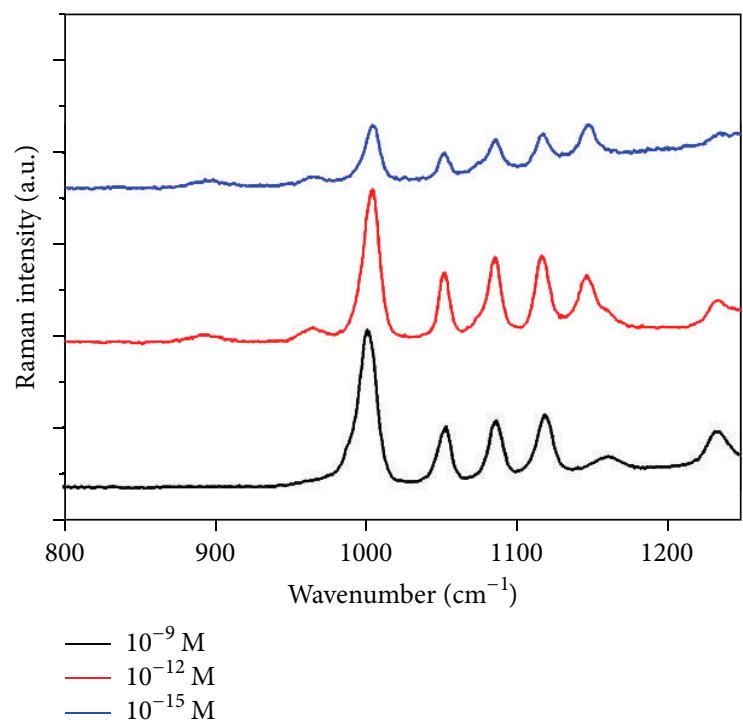

(b)

FIGURE 8: (a) Dark field image of GO/GNRs (1:10). (b) SERS spectra of 2-mercaptopyridine (2-Mpy) with different concentration on the GO/GNRs (1:10): $1 \times 10^{-9}, 1 \times 10^{-12}$, and $1 \times 10^{-15} \mathrm{M}$.

(Figures 7(a) and 7(b)) [27]. GO/GNPs were prepared by the assembly of negatively charged gold nanoparticles $(20 \mathrm{~nm})$ on the surfaces of positively charged polyethylenimine (PEI) modified GO (Figures 7(c) and 7(d)). Curiously, even with 2Mpy at $1 \times 10^{-5} \mathrm{M}$, no Raman bands of 2-Mpy in the range of $900-1200 \mathrm{~nm}$ were observed for both GO/AgNPs and GO/GNPs substrates at the excitation wavelength of $532 \mathrm{~nm}$ (Figures $7(\mathrm{~b})$ and $7(\mathrm{~d})$ ). In contrast, the Raman bands of 2-Mpy on the GO/GNRs are very strong at the excitation wavelength of $785 \mathrm{~nm}$ (Figures 7(e) and 7(f)). The peaks at $1002,1055,1087$, and $1118 \mathrm{~cm}^{-1}$ are assigned to the vibration modes of the 2-Mpy molecule [33]. The results suggested that $\mathrm{GO} / \mathrm{AgNPs}$ and $\mathrm{GO} / \mathrm{GNPs}$ substrates are not suitable for detecting aromatic Raman probes due to the very strong $\pi$ $\pi$ stacking interaction between the aromatic molecules and the $s p^{2}$ carbon structure of GO [34]. For GO/GNRs substrate, the presence of CTAB on GNRs increases the distance of GNRs and GO, thus avoiding the direct touch ( $\pi-\pi$ stacking) between Raman probes and GO. We next investigate the SERS sensitivity of the GO/GNRs. Under the dark field imaging (Figure 8(a)), we can easily find the location of GO/GNRs. As shown in Figure 8(b), the Raman detection limit is as low as $10^{-15} \mathrm{M}$, which is $\sim 2-3$ orders of magnitude higher than that of the corresponding GNRs $\left(\sim 4.5 \times 10^{-12} \mathrm{M}\right)$. The high sensitivity is ascribed to the high density of hotspots on the surface of GO/GNRs.

Several key points are responsible for the design and construction of $\mathrm{GO} / \mathrm{GNR}$ s with ultrahigh density of hotspots. (1) The charge of GO $(-81.33 \pm 3.23)$ and GNRs $(36.09 \pm$ 2.93) directly decides the interaction force between them. (2) The mass ratio of GO and GNRs directly determines sandwich structures: GO-GNRs-GO or GNRs-GO-GNRs. (3) The Raman excitation wavelength should be coupled with the LPSR peak of the inner GNRs.

\section{Conclusions}

In summary, we have developed a convenient and versatile self-assembly strategy of GO and GNRs by means of electrostatic interactions without adding any surfactant, which may be extended to construct other graphene oxide-based assemblies, for SERS application. The as-prepared GO/AgNPs and GO/GNPs substrates are not suitable for detecting 2-Mpy due to the very strong $\pi-\pi$ stacking interaction between the 2 Mpy molecules and the $s p^{2}$ carbon structure of GO. Conversely, the GO/GNRs architecture owns abundant hotspots and exhibits ultrahigh SERS activity and sensitivity of 2-Mpy with the detection limit as low as $\sim 10^{-15} \mathrm{M}$.

\section{Competing Interests}

The authors declare that they have no competing interests.

\section{Acknowledgments}

This work is supported by the National Key Basic Research Program (973 Project) (no. 2015CB931802), National Natural Scientific Fund (nos. 51573096 and 81401465), and 863 Project of China (2014AA020700).

\section{References}

[1] L. Tang, Y. Zhu, L. Xu, X. Yang, and C. Li, "Amperometric glutamate biosensor based on self-assembling glutamate dehydrogenase and dendrimer-encapsulated platinum nanoparticles onto carbon nanotubes," Talanta, vol. 73, no. 3, pp. 438-443, 2007.

[2] Y. Wang, J. Lu, L. Tang, H. Chang, and J. Li, "Graphene oxide amplified electrogenerated chemiluminescence of quantum dots and its selective sensing for glutathione from thiolcontaining compounds," Analytical Chemistry, vol. 81, no. 23, pp. 9710-9715, 2009. 
[3] Z. Nie, A. Petukhova, and E. Kumacheva, "Properties and emerging applications of self-assembled structures made from inorganic nanoparticles," Nature Nanotechnology, vol. 5, no. 1, pp. 15-25, 2010.

[4] Z. Nie, D. Fava, E. Kumacheva, S. Zou, G. C. Walker, and M. Rubinstein, "Self-assembly of metal-polymer analogues of amphiphilic triblock copolymers," Nature Materials, vol. 6, no. 8, pp. 609-614, 2007.

[5] N. Jia, Q. Lian, Z. Tian et al., "Decorating multi-walled carbon nanotubes with quantum dots for construction of multi-color fluorescent nanoprobes," Nanotechnology, vol. 21, no. 4, Article ID 045606, 2010

[6] D. He, B. Hu, Q.-F. Yao, K. Wang, and S.-H. Yu, "Largescale synthesis of flexible free-standing SERS substrates with high sensitivity: electrospun PVA nanofibers embedded with controlled alignment of silver nanoparticles," ACS Nano, vol. 3, no. 12, pp. 3993-4002, 2009.

[7] S. A. Maier, P. G. Kik, H. A. Atwater et al., "Local detection of electromagnetic energy transport below the diffraction limit in metal nanoparticle plasmon waveguides," Nature Materials, vol. 2, no. 4, pp. 229-232, 2003.

[8] J. Lin, S. Wang, P. Huang et al., "Photosensitizer-loaded gold vesicles with strong plasmonic coupling effect for imagingguided photothermal/photodynamic therapy," ACS Nano, vol. 7, no. 6, pp. 5320-5329, 2013.

[9] P. Huang, J. Lin, W. Li et al., "Biodegradable gold nanovesicles with ultra-strong plasmonic coupling effect for photoacoustic imaging and photothermal therapy," Angewandte ChemieInternational Edition, vol. 52, no. 52, pp. 13958-13964, 2013.

[10] X. Liu, J. Wang, Y. Wu et al., "Compact shielding of graphene monolayer leads to extraordinary SERS-active substrate with large-area uniformity and long-term stability," Scientific Reports, vol. 5, Article ID 17167, 2015.

[11] S. Chen, P. Huang, Z. Wang et al., "Self-assembly of gold nanoparticles to silver microspheres as highly efficient 3D SERS substrates," Nanoscale Research Letters, vol. 8, no. 1, article 168, 2013.

[12] S. Nie and S. R. Emory, "Probing single molecules and single nanoparticles by surface-enhanced Raman scattering," Science, vol. 275, no. 5303, pp. 1102-1106, 1997.

[13] X. Qian, X.-H. Peng, D. O. Ansari et al., "In vivo tumor targeting and spectroscopic detection with surface-enhanced Raman nanoparticle tags," Nature Biotechnology, vol. 26, no. 1, pp. 83-90, 2008.

[14] X. Xu, K. Kim, H. Li, and D. L. Fan, "Ordered arrays of raman nanosensors for ultrasensitive and location predictable biochemical detection," Advanced Materials, vol. 24, no. 40, pp. 5457-5463, 2012.

[15] C. G. Khoury and T. Vo-Dinh, "Gold nanostars for surfaceenhanced Raman scattering: synthesis, characterization and optimization," Journal of Physical Chemistry C, vol. 112, no. 48, pp. 18849-18859, 2008.

[16] K. D. Osberg, M. Rycenga, N. Harris et al., "Dispersible gold nanorod dimers with sub-5 nm gaps as local amplifiers for surface-enhanced Raman scattering," Nano Letters, vol. 12, no. 7, pp. 3828-3832, 2012.

[17] K. D. Osberg, M. Rycenga, G. R. Bourret, K. A. Brown, and C. A. Mirkin, "Dispersible surface-enhanced raman scattering nanosheets," Advanced Materials, vol. 24, no. 45, pp. 6065-6070, 2012.

[18] C.-L. Zhang, K.-P. Lv, H.-P. Cong, and S.-H. Yu, "Controlled assemblies of gold nanorods in PVA nanofiber matrix as flexible free-standing SERS substrates by electrospinning," Small, vol. 8, no. 5, pp. 648-653, 2012.

[19] C. Hu, J. Rong, J. Cui et al., "Fabrication of a graphene oxidegold nanorod hybrid material by electrostatic self-assembly for surface-enhanced Raman scattering," Carbon, vol. 51, no. 1, pp. 255-264, 2013.

[20] B. Nikoobakht and M. A. El-Sayed, "Surface-enhanced Raman scattering studies on aggregated gold nanorods," Journal of Physical Chemistry A, vol. 107, no. 18, pp. 3372-3378, 2003.

[21] J. Zhang, H. Yang, G. Shen, P. Cheng, J. Zhang, and S. Guo, "Reduction of graphene oxide vial-ascorbic acid," Chemical Communications, vol. 46, no. 7, pp. 1112-1114, 2010.

[22] P. Huang, C. Xu, J. Lin et al., "Folic acid-conjugated graphene oxide loaded with photosensitizers for targeting photodynamic therapy," Theranostics, vol. 1, pp. 240-250, 2010.

[23] L. Tang, Y. Wang, Y. Li, H. Feng, J. Lu, and J. Li, "Preparation, structure, and electrochemical properties of reduced graphene sheet films," Advanced Functional Materials, vol. 19, no. 17, pp. 2782-2789, 2009.

[24] L. Tang, Y. Wang, Y. Liu, and J. Li, "DNA-directed selfassembly of graphene oxide with applications to ultrasensitive oligonucleotide assay," ACS Nano, vol. 5, no. 5, pp. 3817-3822, 2011.

[25] Q. Zeng, J. Cheng, L. Tang et al., "Self-assembled grapheneenzyme hierarchical nanostructures for electrochemical biosensing," Advanced Functional Materials, vol. 20, no. 19, pp. 3366-3372, 2010.

[26] W. S. Hummers Jr. and R. E. Offeman, "Preparation of graphitic oxide," Journal of the American Chemical Society, vol. 80, no. 6, p. $1339,1958$.

[27] X. Wang, P. Huang, L. Feng et al., "Green controllable synthesis of silver nanomaterials on graphene oxide sheets via spontaneous reduction," RSC Advances, vol. 2, no. 9, pp. 3816-3822, 2012.

[28] J. Zhang, G. Shen, W. Wang, X. Zhou, and S. Guo, "Individual nanocomposite sheets of chemically reduced graphene oxide and poly ( $N$-vinyl pyrrolidone): preparation and humidity sensing characteristics," Journal of Materials Chemistry, vol. 20, no. 48, pp. 10824-10828, 2010.

[29] Z. Li, P. Huang, X. Zhang et al., "RGD-conjugated dendrimermodified gold nanorods for in vivo tumor targeting and photothermal therapy," Molecular Pharmaceutics, vol. 7, no. 1, pp. 94-104, 2009.

[30] P. Huang, L. Bao, C. Zhang et al., "Folic acid-conjugated silicamodified gold nanorods for X-ray/CT imaging-guided dualmode radiation and photo-thermal therapy," Biomaterials, vol. 32, no. 36, pp. 9796-9809, 2011.

[31] B. Tian, C. Wang, S. Zhang, L. Feng, and Z. Liu, "Photothermally enhanced photodynamic therapy delivered by nanographene oxide," ACS Nano, vol. 5, no. 9, pp. 7000-7009, 2011.

[32] X. Fu, L. Chen, J. Li, M. Lin, H. You, and W. Wang, "Labelfree colorimetric sensor for ultrasensitive detection of heparin based on color quenching of gold nanorods by graphene oxide," Biosensors \& Bioelectronics, vol. 34, no. 1, pp. 227-231, 2012.

[33] D.-P. Yang, S. Chen, P. Huang et al., "Bacteria-template synthesized silver microspheres with hollow and porous structures as excellent SERS substrate," Green Chemistry, vol. 12, no. 11, pp. 2038-2042, 2010.

[34] J. Huang, L. Zhang, B. Chen et al., "Nanocomposites of sizecontrolled gold nanoparticles and graphene oxide: formation and applications in SERS and catalysis," Nanoscale, vol. 2, no. 12, pp. 2733-2738, 2010. 

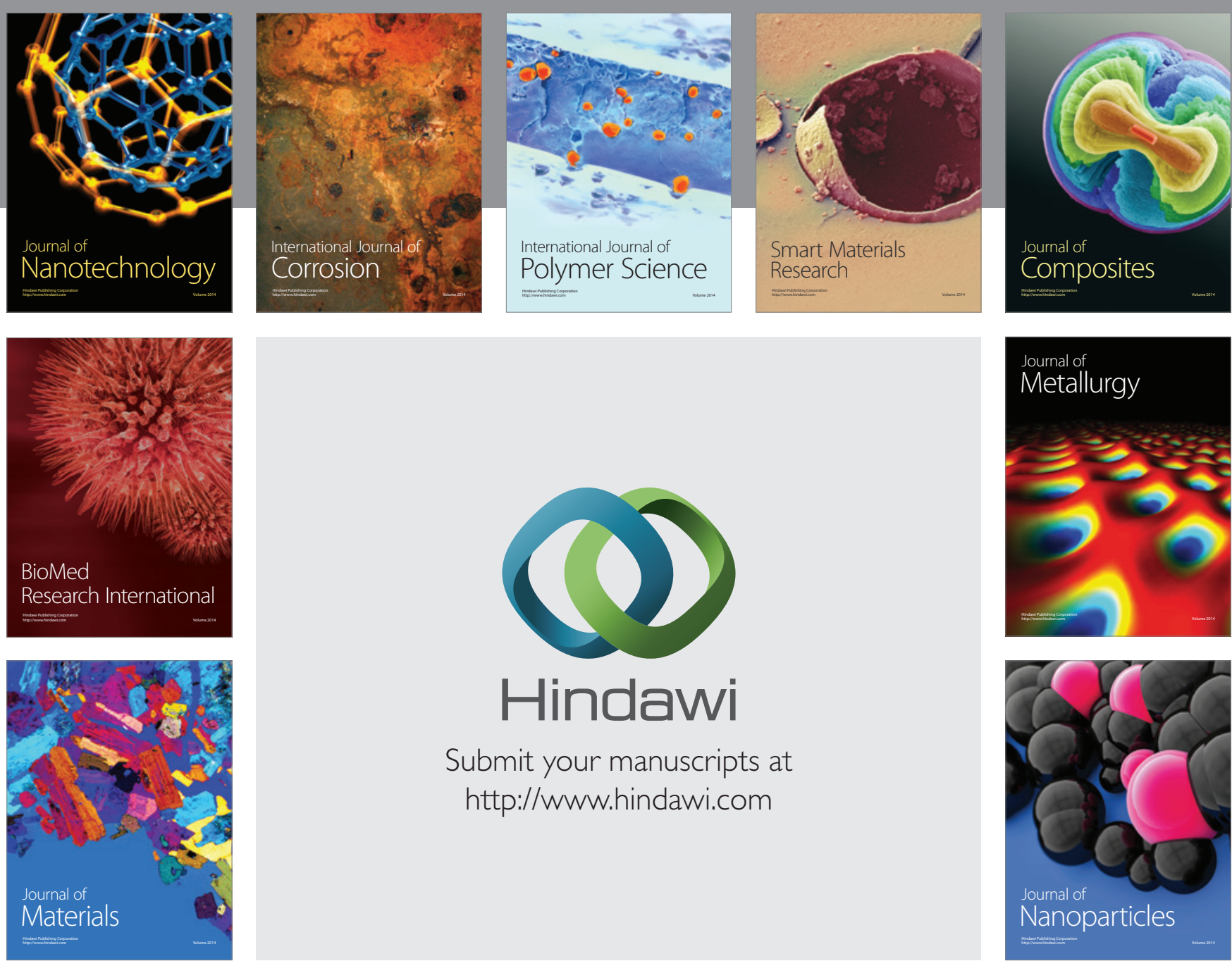

\section{Hindawi}

Submit your manuscripts at

http://www.hindawi.com

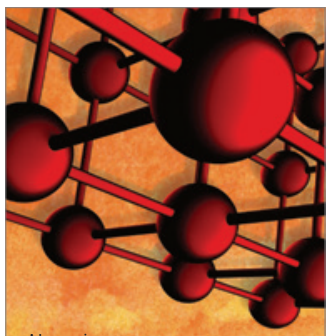

Materials Science and Engineering
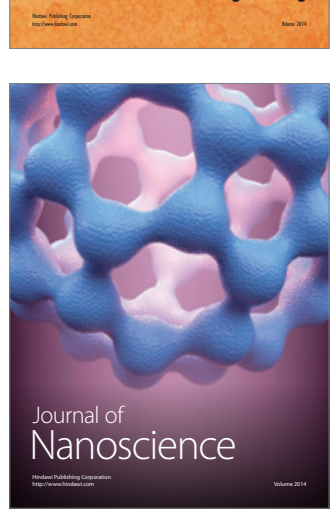
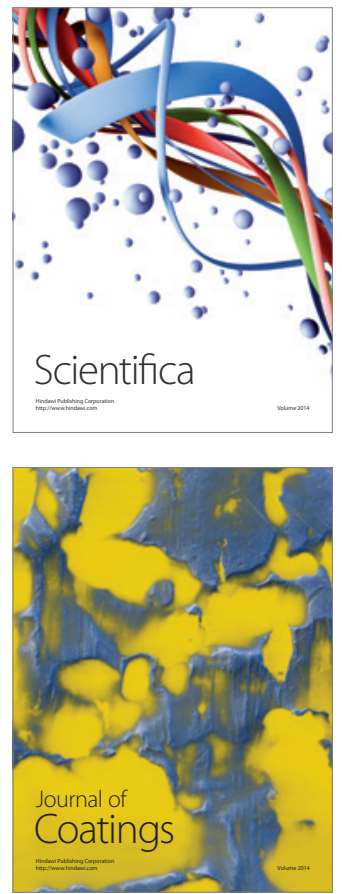
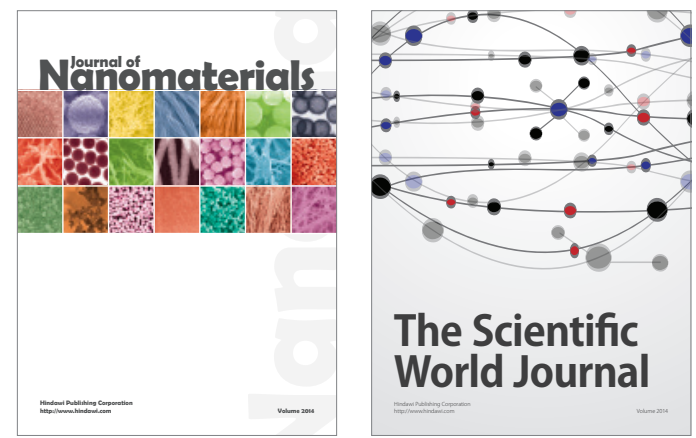

The Scientific World Journal
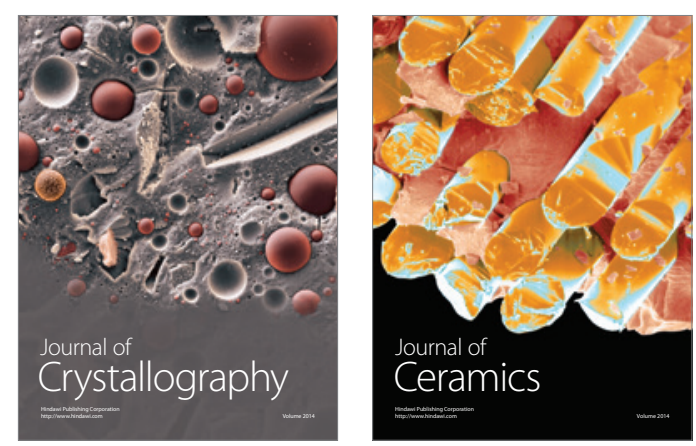
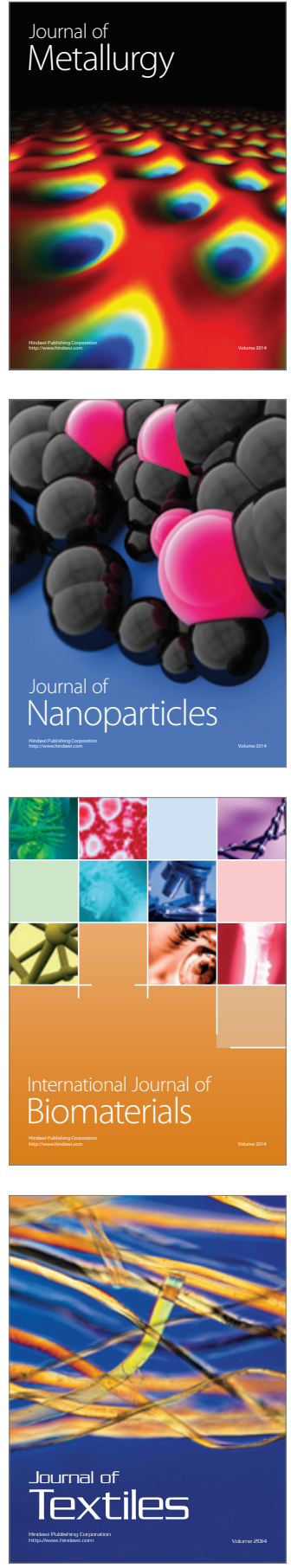